\title{
Osteosarcoma of limb bones: a clinical, radiological and histopathological diagnostic agreement at Black Lion Teaching Hospital, Ethiopia
}

\author{
Biruk L Wamisho ${ }^{1}$, Daniel Admasie ${ }^{2}$, Bayush E Negash ${ }^{3}$, Mihiret W Tinsay ${ }^{4}$
}

1. Beit Cure International Hospital, Malawi

2. Addis Ababa University, Medical Faculty (AAU-MF), Ethiopia

3. Radiology, Addis Ababa, Ethiopia

4. Dept of Pathology, Addis Ababa University, Ethiopia

Correspondence: Dr. Biruk L. WAMISHO, e-mail: lbiruklw@yahoo.com

Tel: (+00251)911232507; P.O.Box: 122201, Addis Ababa, Ethiopia

\section{Abstract}

Objectives: To measure the strength of agreement in clinical, radiological and histopathological diagnosis of osteosarcoma in a 5 year study period.

Setting: Addis Ababa University, Black-Lion ('Tikur Anbessa') Hospital-BLH, is the country's highest tertiary level referral and teaching hospital. The departments involved in this study (Radiology, Pathology and Orthopedics) receive referred patients from all over the country.

Methods: All bone tumor patients, presenting to the three departments at BLH between the study period, December, 2003 - March, 2008 were recruited for the study. 51 patients with radiological diagnosis of osteosarcoma of the extremities were identified and their clinical and histopathological diagnoses reviewed in detail. All patients had a clinical examination, plain radiographs and biopsies of the affected part of the extremity. Radiographs of selected difficult cases were discussed at joint orthopedic \& radiologic sessions every week. The radiological and histopathological diagnoses made were categorized separately using WHO classification of bone tumors. Strength of agreement between radiological and histopathological diagnoses was measured using Cohen's Kappa test.

Results: Of the total of 216 bone tumor patients presented and biopsied in the five year period, fifty one (51) had osteosarcoma of extremity bones. Commonest age affected by osteosarcoma was 16 (7-55years) and sex ratio was 1:1. Osteosarcoma was also the single most common clinical, radiological and histological diagnosis made. Considering all bone tumors presented together, the study indicated that radiological diagnosis was confirmed by similar histological diagnosis in 172 out 205 cases $(84 \%)$ and the corresponding Cohen's Kappa value (0.82) showed excellent level of agreement between radiological and histological diagnoses of all bone tumors. The agreement between radiological and histopathological diagnoses of osteosarcoma of the limbs was $84.5 \%$.

Conclusion: There is an excellent agreement between clinical, radiological and histopathological diagnoses of bone tumors in general and osteosarcoma in particular. In setups where there is limited or no histopathological service, joint clinical and radiological decision could lead to a higher degree of accuracy in diagnosis of bone tumors, especially osteosarcoma. Radiological diagnosis of bone tumors at Black Lion Hospital, Addis Ababa is excellent.

\section{Introduction}

Osteosarcoma is a primary malignant tumour of bones that has been found to be more common in males than females. It has been reported to be the commonest primary malignant bone tumor. A review of literature reveals that Osteosarcoma-like lesions were demonstrated in the femur and humerii of ancient Egyptian mummies ${ }^{1}$. For a long time, osteosarcoma has been associated with a low social-economic status ${ }^{2}$. This is a major problem in the developing countries where limbsparing surgery is not yet possible. Amputation is therefore often the mode of treatment making the patient disabled, non productive and a burden to the family $3,4,5$.

In a study carried out in Uganda between 1964 and 1968, osteosarcoma was found to be the commonest primary malignant bone tumour with a peak age of $10-19$ years ${ }^{2}$. The commonest site was the lower femur ${ }^{2}$. This is similar to what has been reported elsewhere ${ }^{6}$. It has been suggested that major histocompatibility complex linked genes may determine susceptibility to osteosarcoma ${ }^{7}$. Other possible epidemiological factors suggested are mechanical trauma, ionizing radiation and chronic osteomyelitis ${ }^{8-12}$.

Radiological diagnosis takes into account the site of lesion, borders of the lesion, type of matrix, type of bone destruction, type of periosteal reaction, nature and extent of soft tissue involvement and number of lesions. Radiologically, osteogenic sarcoma presents commonly around the knee joint and is metadiaphyseal. It presents as an eccentric area of bone destruction with cortical erosion and a soft tissue mass. At scintigraphy, it may show increased vascularity. Other investigative modalities of value in osteosarcoma are computerized tomography, magnetic resonance and angiography. A systematic approach to clinical history, radiographic evaluation and histopathology is necessary for accurate diagnosis of osteosarcoma-all three are important. As many bone lesions overlap, an experienced clinician systematically integrates the radiological \& histopathological results to plan \& offer the best possible management to the patient.

\section{Patients and methods}

All bone tumor patients, who presented to BLH between the study period, December, 2003 - March, 2008 were entered into the study. 51 patients with radiological diagnosis of osteosarcoma in any of the limb bones were identified and their clinical and histological diagnosis looked at in detail. Hematologic tumors including malignant myeloma were excluded from the study, as they are mainly diagnosed by bone marrow cytology and not bone tissue biopsy. All files related to these patients were reviewed. All patients had a clinical examination, plain radiographs and biopsies of the affected part of the extremity. Radiographs of selected difficult cases were discussed at joint orthopedic \& radiologic weekly sessions. The radiological and histopathological diagnoses 
made were categorized separately using WHO classification of bone tumors ${ }^{13}$. Chest $\mathrm{x}$-rays, ultrasound examination and organ function tests were performed in selected patients to rule-out metastases. The clinical history, radiological and histological data was then retrieved. The data was analyzed using SPSS and strength of agreement in diagnosis was calculated using Cohen's Kappa test online ${ }^{14}$.

\section{Results}

Of the total of 216 bone tumor patients presenting in the five year period, fifty one (51) patients with osteosarcoma of the extremity bones were reviewed in detail in this study. Complete information could not be gathered from $11(5 \%)$ of the cases and these are excluded. Over all, there were $111(51.3 \%)$ males and $94(48.7 \%)$ females with histopathological tissue diagnosis of bone tumor. Male to female ratio was nearly $1: 1$. There were 32 different types of bone tumors. $11.2 \%(23 / 205)$ of the tumors were nonneoplastic and $5 \%(10 / 205)$ of all the tumors were metastatic lesions. $36 \%,(74 / 205)$ of the tumors were malignant. Primary bone tumors accounted for $95 \%$ (172/182) of all bone neoplasms. The commonest primary malignant tumor diagnosed by bone biopsy was osteosarcoma and commonest age affected was 16 years (Age range 7-55 years), figure 1. Most $(87 \%)$ extremity osteosarcomas occurred in patients under 30 years of age. Four were aged over 35 . Osteosarcoma was also the single most common clinical, radiological and histological diagnosis made. Exostosis was second.

The presenting complaints included pain in the affected limb, swelling, tenderness and venous engorgement. Osteosarcomas of other bones or tissues were excluded. As shown on figure 2, the commonest complaints in osteosarcoma were pain $(92 \%)$ and a growing swelling (87\%). Long bones of the extremities were involved in $90 \%(47 / 52)$ of all histopathologically diagnosed cases of osteosarcoma of bones. Four patients $(7.8 \%)$ had primary pelvic osteosarcoma, table 1. Excluding parosteal osteosarcoma, in all proven lesions, average duration of illness at presentation was 4-5 months. On average, seven weeks (7days to 5 months) elapsed between
Table 1 Distribution of the Clinical site of Osteosarcoma in biopsied bone tumors of the Limbs at BLH, Dec. 2003-Mat. 2008

\begin{tabular}{|l|l|l|}
\hline Site of the Bone Tumor & Number & Percent \\
\hline Distal Femur & 25 & 49 \\
\hline Proximal Tibia & 9 & 17.7 \\
\hline Proximal Humerus & 6 & 11.8 \\
\hline Proximal Femur & 3 & 5.9 \\
\hline Proximal Fibula & 2 & 3.9 \\
\hline Distal Tibia & 1 & 1.9 \\
\hline Proximal Phalanx & 1 & 1.9 \\
\hline *Pelvic Bones & 4 & 7.9 \\
\hline Total & $\mathbf{5 1}$ & $\mathbf{1 0 0}$ \\
\hline
\end{tabular}

*The new WHO Classification has a separate category for pelvic bone tumors. There is only 1 additional maxillary tumor which is biopsied.

initial presentation to the hospital and undergoing biopsies. One lesion was diaphysial and occurred in the femur while the rest were metadiaphyseal. Two patients presented with pathological fractures. All long bone lesions involved the cortex and medulla of bone and most $(60 \%, 28 / 47)$ were of mixed density, 15 were sclerotic and 4 was completely lytic. All were solitary lesions with a wide zone of transition. All had a periosteal reaction. About half $(49 \%, 25 / 51)$ had a Codman's triangle. Other types of periosteal reaction included sunburst (10) and lamellated (8).

All but five of the lesions extended to the soft tissues with preservation of fat planes. Parosteal lesion was seen only in three patients. Figures $1-2$ show typical radiological appearance of osteosarcoma Histopathologically proven osteosarcoma accounted for $88.2 \%(45 / 51)$ of all osteogenic tumors. Considering all bone tumors together, the study indicated that radiological diagnosis was confirmed by histological diagnosis in 172 out 205 cases (84\%) and the corresponding Cohen's kappa value (0.82) showed excellent agreement between radiological and histological diagnoses of all bone tumors. The agreement between radiological and histological diagnosis of osteosarcoma of the limbs was

Fig. 1 Age distribution of osteosarcoma, Addis Ababa University, BLH. Dec. 2003-Mar. 2008

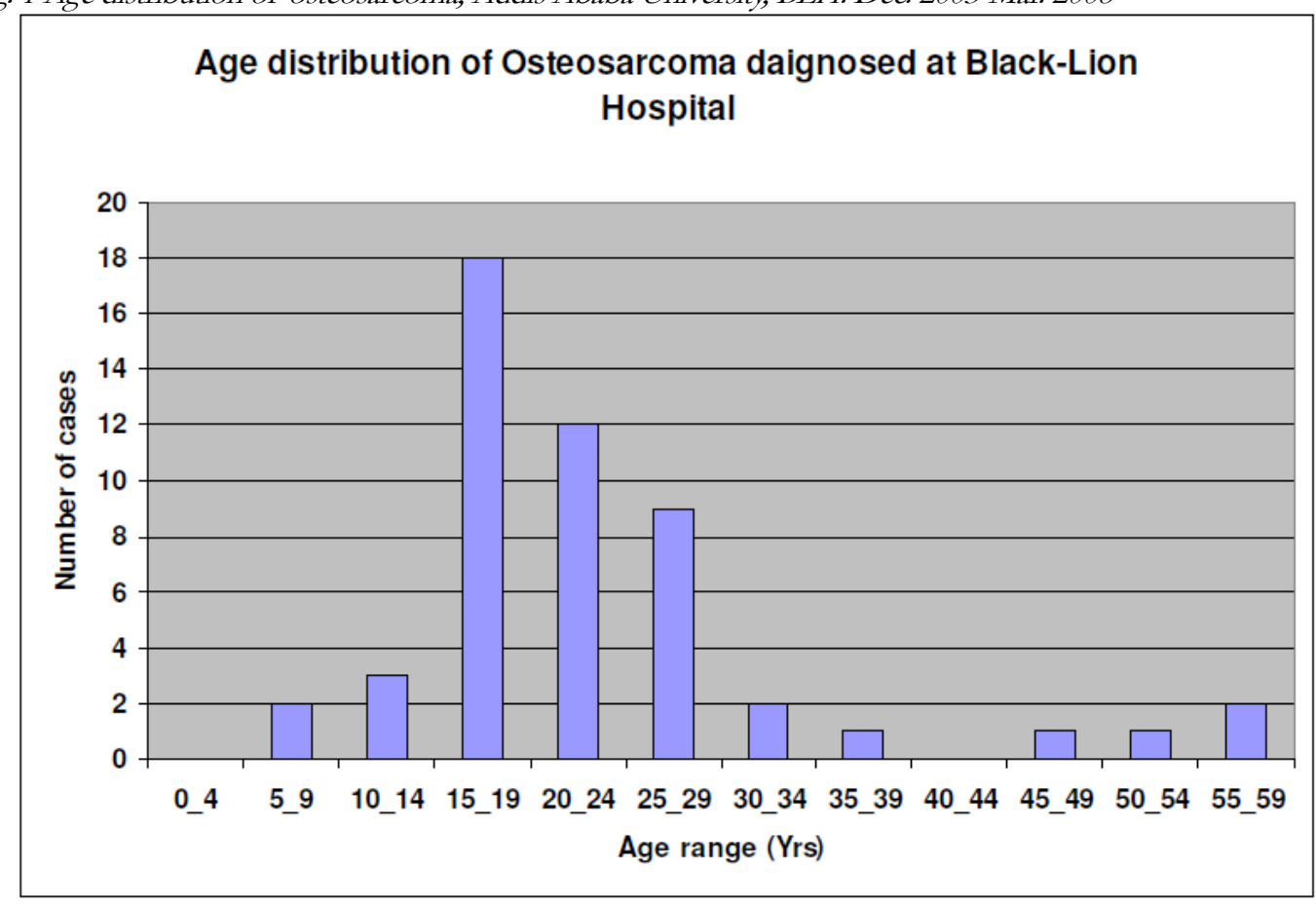




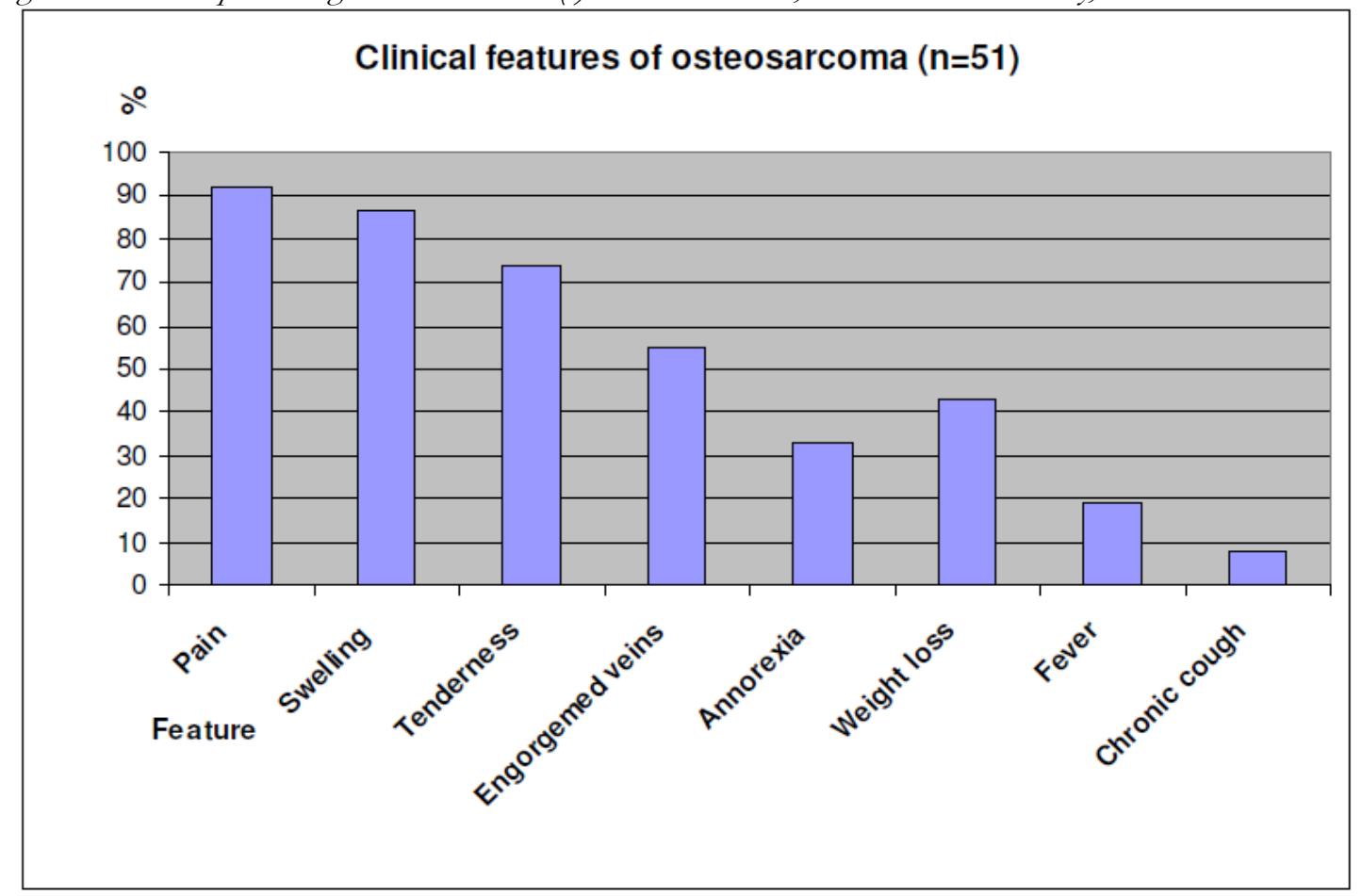

$84.7 \%$. Radiological and histological diagnosis were not in agreement in eight $(15.3 \%, 8 / 52)$ of the patients. $11 \%$ of cases with a histological diagnosis of a benign tumour were diagnosed as malignant of $\mathrm{x}$-ray; these are shown on table 2.

Table 2: Disagteements between radiolooical and histopathological giagnosis of osteosarcoma of the limbs at BLH, Dec. 2003-Mat. 2008

\begin{tabular}{|l|l|l|}
\hline $\begin{array}{l}\text { Radiological } \\
\text { diagnosis }\end{array}$ & Pathological Diagnosis & Frequency \\
\hline Osteosarcoma & ${ }^{*} \mathrm{MFH}$ & 2 \\
\hline Osteosarcoma & \#GCT & 2 \\
\hline Lymphoma & Osteosarcoma & 1 \\
\hline Osteosarcoma & Lieomyosarcoma & 1 \\
\hline Osteosarcoma & Ossifying fibroma & 1 \\
\hline Osteosarcoma & Fibrous dysplasia & 1 \\
\hline
\end{tabular}

* MFH=Malignant Fibrous Histiocytoma, \#GCT= Giant Cell Tumor.

\section{Discussion}

Primary bone tumors are rare and account only for $0.2 \%$ of human tumors ${ }^{15}$. Osteosarcoma has been reported to be the commonest primary malignant bone tumour. In our study, it was commonest in the age range 15 - 29 years, akin to the text book description of $10-25$ years. The sex and site distribution of osteosarcoma in our patients is also comparable and very similar to other East African studies ${ }^{2,16 \text {, }}$ 17, 18. About three fourths of the tumor occurred around the knee joint. The increased incidence of osteosarcoma during this age range has been attributed to the preceding increased growth spurt of adolescence.

We have noted that the documentation of bone tumor patients at Black-Lion Hospital is excellent, only $5 \%$ $(11 / 216)$ files were lost. This could be due to the fact that the analysis focused only on the last five years, which are very recent. We also noted that, the Pathology Department has made a spectrum of 32 different types of histopathological bone lesions, of which osteosarcoma is one. This shows the

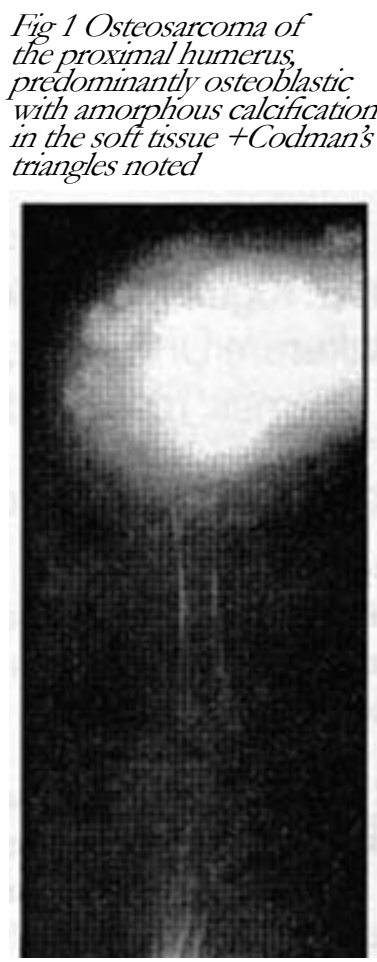

Fig 2. Osteosarcoma of the proximal tibia, predominantly. osteoblastic, cortical destruction and periosteal reaction

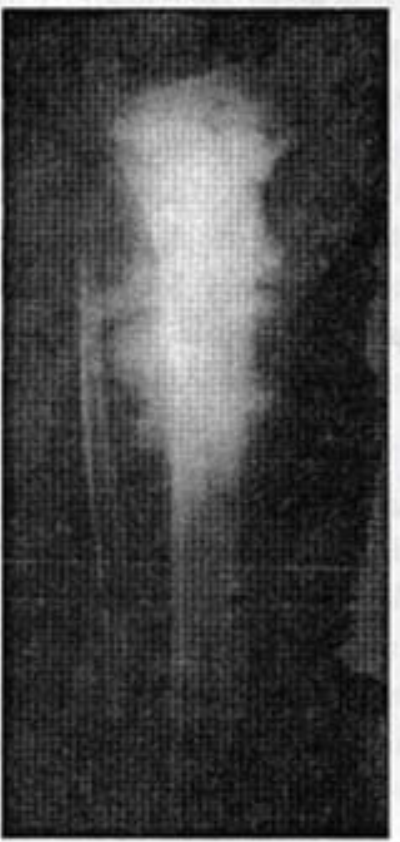

Departments long years of experience in detecting different bone lesions and it also indicates the presence of many types of bone tumors in a referral hospital.

In our study, only $9.4 \%(4 / 51)$ of the patients were over 35 years but none had a premalignant conditions like Paget's disease, multiple Exostosis, fibrous dysplasia or had had radiation therapy. These benign conditions have been reported to undergo transformation to malignancy ${ }^{19}$. The female to male ratio in this study was $1: 1$, similar to the Ugandan series ${ }^{2}$. Pain was the commonest presenting symptom and this is similar to what has been reported in other series, this was followed by swelling ${ }^{20,21}$.

Our patients presented very late to the hospital, these could be partly due to poor referral systems. Patients also have to 
wait for unacceptable long periods of time for admission tissue biopsy and subsequent management; this may be due to the limited number of orthopedic beds available at Black Lion Hospital. It could also be due to the overwhelming number of trauma patients competing for few beds. An easy way to solve this could be to do bone biopsy as a day-case surgery in out patients.

In this series the distal third of femur was the most common site affected like many other studies ${ }^{22}$. Osteosarcoma has been noted to be rare in the axial skeleton, it was found in the pelvic skeleton only in $4(9.4 \%)$ of our patients ${ }^{23}$. Kappa test -statistic used to measure the level/strength of agreement between different raters (orthopedists, radiologists and pathologists) in placing/diagnosing bone tumors into the different WHO categories was calculated online and had a value of $82 \%(0.82)$. This is an excellent level of agreement $(\mathrm{k}>0.75)$ between radiological and histological diagnoses of all bone tumors ${ }^{24}$. However there are still some tumours that are diagnosed to be malignant on $\mathrm{x}$-ray but are later proven to be benign. If the diagnosis is not confirmed by histology then there is a risk of inappropriate surgery being carried out. Therefore bone tumor diagnosis should be made by joint consideration of clinical presentation, radiological appearance and histopathological results whenever possible.

\section{Conclusion}

There is an excellent agreement between clinical, radiological and histological diagnosis of bone tumors in general and osteosarcoma in particular. In set-ups where there is limited or no histopathological service, joint clinical and radiological decision could lead to a higher degree of accuracy in diagnosis of bone tumors, especially osteosarcoma. Radiological diagnosis of bone tumors at Black Lion Hospital, Addis Ababa is excellent.

\section{Recommendations}

Based on the findings of this study, we recommend the following:

- As exact diagnosis of bone tumors is at times difficult, a joint approach integrating clinical, radiological and histopathological findings is recommended to increase accuracy. This is a good lesson from BLH.

- Where there is no or limited histopathological service, the surgeon could decide on the patient's management based on radiologist's report and clinical findings, but risks misdiagnosing some benign tumours as malignant. Wherever available, histopathological diagnosis should help the surgeon in planning limb-salvaging surgery for early malignant and all benign bone lesions.
- Patients with osteosarcoma have present to the hospital very late (average 4-5 months) after onset of symptoms. Increased awareness and improved referral systems may decrease this delay and limb-salvaging operations may be possible.

- Mechanisms to decrease the time waiting for biopsies should be sought as osteosarcoma requires urgent attention. This could be achieved partly by increasing the number of orthopedic beds and theatre time in the hospital.

\section{Acknowledgments}

We thank the department of Radiology and Pathology in the Medical faculty for providing access to patient information. We appreciate our patients' co-operation in the study. Thank you Lili for giving us this idea of group-work on bone tumors.

\section{References}

1. Hamada G, Rida A. Orthopaedics and Orthopaedic diseases in Ancient and Modern Egypt: Clin Orthop. 1972; 89: 253.

2. Dodge O.D. Tumours of bone and jaw. In: Templeton AC, (Ed). Turnouts in a Tropical country. Springer - Verlag Berlin - Heidelberg. 1973; 14: 222 - 233.

3. Sebaggala S.J.M. The clinical presentations of patients with Osteosarcoma in Mulago Hospital 1987- Jan 1997. M Med.Thesis 1998, 1:1 -2, 2:4-10.

4. Ahuka OL, Lusi KM. Amputations in rural areas of another Zaire: an aetiological and epidemiological study. East and Central Afr J Surg $1995 ; 1: 33-35$.

5. Kiwanuka M. Lower limb amputation in Uganda. M Med Thesis 1983 May; 3:40.

6. Parkin, Whelan, Raymond, Young: Cancer incidence in five Continents IARC scientific publications Vol. 7 No. 143 1997; P 70, 78-86.

7. Turo S, Masaki C, Mitsuo N, Nideomi W, Eiichi U HLA Phenotypes in patients who have Osteosarcoma. J. Bone Joint Surg 1990; 72A: 68.

8. Fornasier VL, Protzner K. Radium induced tibial sarcoma in a treated case of hind foot angiomatosis: Skeletal Radiology. Vol. 27 No. 31998 Mar; P 164-8.

9. Bascoulergue G, Gorgeon F, Lecomte -Houcke M, Baviera E, Mazabraud M. Malignant synovial chondromatosis or chondromatous transformation of synovial chondromatosis of the knee: Bulletin du cancer. Vol 83 No. 111996 Nov; P951 -6.

10. Hasbini A, Lartigau E, Le Pechoux C, Acharki A, Vanel D, GeninJ, Le Cesne A. Chondrosarcoma in Ollier's disease: a propos of two cases and review of Literature. Cancer Radiotherapie. Vol.2 no. 4 1998Ju1-Aug; P384-91.

11. Adil A, Hoeffel, Fikry T. Osteoid Osteoma After a fracture of the radius. AJR 1996; 167:145 - 6.

12. Maeda G, Yokoyama R, Othomo K, TakayamaJ, Beppu Y, Fukuma $\mathrm{H}$, Ohira M. Osteochondroma after total body irradiation in bone marrow transplant patients: Report of 2 cases (Review) (14 Refs), Japanese article of clinical oncology. 1996; 6: 480 -3.

13. Dorfman H D, Czerniak B, and Kotz R. WHO classification of tumors bone:. In WHO,Classification of tumors of soft tissue and bone, pages 227 - 232. Lyon: IARC press, 2002. 